\title{
Achados endoscópicos em portadores de sintomas dispépticos
}

\section{Endoscopic findlings in patients with dyspeptic symptoms \\ Hallazgos endoscópicos en pacientes con síntomas dispépticos}

Kellyn Mariane Souza Sales

ORCID: https://orcid.org/0000-0001-6233-5103

Universidade Tiradentes, Brasil

E-mail: kellynsales1@gmail.com

Wianne Santos Silva

ORCID: https://orcid.org/0000-0001-8006-8934

Universidade Tiradentes, Brasil

E-mail:wiannessilva@gmail.com

Gabriel Ponciano Santos de Carvalho

ORCID: https://orcid.org/0000-0001-7476-7907 Universidade Tiradentes, Brasil

E-mail: gabriel.ponciano19@gmail.com

Anna Marcela Lima Fonseca

ORCID: https://orcid.org/0000-0001-5519-965X Universidade Tiradentes, Brasil

E-mail: marcela.lima0102@gmail.com

Giovanna Pimentel Oliveira Silva

ORCID: https://orcid.org/0000-0002-4981-8466 Universidade Tiradentes, Brasil

E-mail: giovannapimente196@gmail.com

Thaissa Carvalho Viaggi

ORCID: https://orcid.org/0000-0002-0026-7149

Universidade Tiradentes, Brasil

E-mail: thaissaviaggi@gmail.com

Beatriz Carvalho Aragão

ORCID: https://orcid.org/0000-0002-2805-9675

Universidade Tiradentes, Brasil

E-mail: bilaaragaoc@gmail.com

Ana Monize Ribeiro Fonseca

ORCID: https://orcid.org/0000-0002-7565-8435 Universidade Tiradentes, Brasil

E-mail: anamonizerf@gmail.com

Allef Francisco Lira da Rocha Braga

ORCID: https://orcid.org/0000-0001-7326-0012

Universidade Tiradentes, Brasil

E-mail: allefbraga@gmail.com

Arthur Matos Bitencourt Chagas

ORCID: https://orcid.org/0000-0003-1276-3358 Universidade Tiradentes, Brasil

E-mail: arthurmatos95@gmail.com

Beatriz Mariana de Andrade Guimarães ORCID: https://orcid.org/0000-0002-7792-3810 Universidade Tiradentes, Brasil

E-mail: biaguimaraees@outlook.com

Leda Maria Delmondes Freitas Trindade ORCID: https://orcid.org/0000-0003-4300-4274

Universidade Tiradentes, Brasil

E-mail: ledeltrin@gmail.com

Frederico Santana de Lima

ORCID: https://orcid.org/0000-0002-5251-5976

Universidade Tiradentes, Brasil

E-mail: fredericogastro@gmail.com

\section{Resumo}

Introdução: A endoscopia tem importante valor para avaliar as queixas dispépticas. É sabido que os achados vistos ao exame conseguem determinar a presença de doenças estruturais que justifiquem tais sintomas. Objetivo: Identificar a prevalência de achados endoscópicos em pacientes portadores de sintomas dispépticos. Metodologia: Estudo prospectivo, transversal e descritivo realizado na cidade de Aracaju/SE, em pacientes que realizaram EDA e tinham diagnóstico prévio de dispepsia ou queixas dispépticas. Foram utilizadas amostras de 859 laudos de indivíduos com 
diagnóstico de dispepsia e aplicados questionários do tipo sociodemográfico e clínico com questões abertas e fechadas, além de questionário contendo os Critérios de ROMA III. Ao final, 847 foram considerados válidos, 792 (93,5\%) tinham alguma alteração, 36 (4,5\%) laudos foram considerados normais, ou seja, sem lesão estrutural e $19(2,39 \%)$ deles não foram liberados pelos pacientes. A faixa etária mediana (IIQ) foi de 37 (30 a 46) anos, 549 (64,8\%) eram do sexo feminino e $617(72,8 \%)$ tinham procedência da capital. Dentre os achados endoscópicos foi estatisticamente significativo o diagnóstico de gastrite $(\mathrm{p}<0,001)$, com $661(89,2)$ casos. Foram mais prevalentes percentualmente os laudos com diagnóstico de esofagite $168(22,7 \%)$ e de pólipo $54(6,4 \%)$. Dos 54 pacientes que apresentaram pólipos, $49(90,7 \%)$ eram do tipo gástrico, 4 (6,3\%) esofágicos e $3(3,1 \%)$ duodenais. Conclusão: A maioria dos pacientes com queixas dispépticas apresentaram alterações estruturais vistas à EDA que justificaram a presença dos sintomas.

Palavras-chave: Dispepsia; Endoscopia; Sintomas.

\begin{abstract}
Introduction: Endoscopy has an important value to assess dyspeptic complaints. It is known that the findings seen on examination can determine the presence of structural diseases that justify such symptoms. Objective: Identify the prevalence of endoscopic findings in patients with dyspeptic symptoms. Methodology: Prospective, cross-sectional and descriptive study conducted in the city of Aracaju / SE, in patients who underwent EGD and a previous clinician of dyspepsia or dyspeptic complaints. Samples of 859 reports from individuals diagnosed with dyspepsia were used and sociodemographic and clinical questionnaires with open and closed questions were applied, as well as a questionnaire containing the ROMA III criteria. In the end, 847 were considered valid, $792(93.5 \%)$ had some alteration, $36(4.5 \%)$ reports were considered normal, that is, without structural damage and $19(2.39 \%)$ of them did not were released by the patients. The median age group (IIQ) was 37 (30 to 46) years, $549(64.8 \%)$ were female, 617 (72.8\%) were from the capital. Among the endoscopic findings, the diagnosis of gastritis was statistically significant $(\mathrm{p}<0.001)$, with 661 (89.2) cases. The most prevalent percentages were the reports with the diagnosis of esophagitis $168(22.7 \%)$ and polyp 54 (6.4\%). Of the 54 patients who had polyps, 49 (90.7\%) were gastric, 4 (6.3\%) esophageal and $3(3.1 \%)$ duodenal. Conclusion: Most patients with dyspeptic complaints had structural changes seen in the EGD that justified the presence of symptoms.
\end{abstract}

Keywords: Dyspepsia; Endoscopy; Symptom.

\title{
Resumen
}

Introducción: La endoscopia tiene un valor importante para evaluar las molestias dispépticas. Se sabe que los hallazgos observados en el examen pueden determinar la presencia de enfermedades estructurales que justifiquen tales síntomas. Objetivo: identificar la prevalencia de hallazgos endoscópicos en pacientes con síntomas dispépticos. Metodología: estudio prospectivo, transversal y descriptivo realizado en la ciudad de Aracaju / SE, en pacientes sometidos a EGD y con diagnóstico previo de dispepsia o quejas dispépticas. Se utilizaron muestras de 859 informes de personas diagnosticadas con dispepsia y se aplicaron cuestionarios sociodemográficos y clínicos con preguntas abiertas y cerradas, así como un cuestionario que contenía los criterios ROMA III. Al final, de los 859 informes, 847 se consideraron válidos, 792 (93,5\%) tenían alguna alteración, 36 (4,5\%) informes se consideraron normales, es decir, sin daño estructural y $19(2,39 \%)$ de ellos no lo fueron liberado por los pacientes. El grupo de edad promedio (IIQ) fue de 37 (30 a 46) años, 549 (64,8\%) eran mujeres, 617 (72,8\%) eran de la capital. Entre los hallazgos endoscópicos, el diagnóstico de gastritis fue estadísticamente significativo ( $\mathrm{p}<0,001)$, con $661(89,2)$ casos. Los porcentajes más prevalentes fueron los reportes con diagnóstico de esofagitis $168(22,7 \%)$ y pólipo $54(6,4 \%)$. De los 54 pacientes que tenían pólipos, 49 (90,7\%) eran gástricos, 4 (6,3\%) esofágicos y $3(3,1 \%)$ duodenales. Conclusión: La mayoría de los pacientes con quejas dispépticas presentaron cambios estructurales observados en la EGD que justificaron la presencia de síntomas.

Palabras clave: Dispepsia; Endoscopía; Síntomas.

\section{Introdução}

A dispepsia ou síndrome dispéptica faz parte das queixas mais comuns relacionadas ao trato digestivo no cenário clínico atual, com ocorrência em pelo menos $20 \%$ da população (Longstreth, Lacy, Talley, \& Grover, 2013). É uma condição complexa, incluindo o trato gastrointestinal superior com sintomas crônicos e recorrentes, como dor epigástrica, desconforto, plenitude pósprandial e saciedade precoce, que podem se sobrepor a azia e regurgitação (Silva, 2008).

Pacientes dispépticos podem apresentar vários sintomas com variação deles no mesmo paciente ao longo do tempo. O dilema está em compreender e quantificar o que os pacientes estão tentando dizer aos médicos. O que eles querem dizer quando se queixam de indigestão e o que os médicos procuram transmitir ao usar o termo dispepsia? (Koduru, Irani, \& Quigley, 2018).

A queixa de um único sintoma é incomum. O que normalmente se observa é a apresentação de vários sintomas em conjunto, sendo que alguns trabalhos demonstram média de até quatro sintomas por paciente (Magalhães, de Moraes, \& Braga, 
M., 2018). Tais sintomas refletem em suas relações pessoais, sociais e laborais, ocasionando alterações psicológicas, do sono e da atividade sexual (Matsuda, Maia, \& Troncon, 2010). Desta forma, a qualidade de vida de forma é reduzida de forma considerável e mesmo que a maioria deles não procure atendimento médico inicial, uma proporção considerável de pacientes o fará durante o curso de sua doença, causando grande impacto nos custos de saúde e baixo rendimento da endoscopia (Pineda et al, 2015).

Os sintomas de dispepsia podem ocorrer devido a um importante fator estrutural, como úlcera crônica gastroesofágica ou também pode se apresentar como dispepsia funcional, que significa sem evidência de causa orgânica (Chehreh, Shahverdi, Khedmat, Ghafoorian, \& Amini, 2016). Assim, é um agrupamento de sintomas resultantes de distintas doenças, sendo estudada a partir das mesmas (Oliveira, 2001).

O rendimento da endoscopia em pacientes com dispepsia é questionável e varia entre os estudos; parte desta variação é devido a diferentes definições de dispepsia usadas por diferentes estudos (Ford, Marwaha, Sood, \& Moayyedi, 2015). A endoscopia é o padrão ouro na avaliação de pacientes dispépticos e várias diretrizes foram elaboradas para tornar o uso da endoscopia digestiva alta mais racional (Akram, Mohammed, Mohammed, \& Obaid, 2019). Desta maneira, quando utilizado de forma correta pode trazer dados expressivos e importantes quanto às principais patologias encontradas em pacientes dispépticos, podendo ter como causas: úlceras pépticas, refluxo gastroesofágico, medicamentos (agentes anti-inflamatórios não esteróides sendo o agressor mais comum), malignidade gástrica, dentre outros. Faltam pesquisas com bases científicas para sistematizar quais pacientes devem ser submetidos à endoscopia e quais diagnósticos são observados nas diferentes faixas etárias (Fonseca, D'Acampora, Zeni, Sombrio, \& Oliveira, 2014).

Logo, esta pesquisa teve como proposta analisar a prevalência dos achados endoscópicos em portadores de sintomas dispépticos em duas clínicas e dessa forma auxiliar para formação de novos dados, contribuindo com a pesquisa no Estado de Sergipe.

\section{Metodologia}

Estudo prospectivo, transversal e descritivo, realizado no período de 2018 a 2019, em duas clínicas privadas de endoscopia digestiva (EDA), em Aracaju, Sergipe. Amostra tipo probabilística por conveniência foi composta por 859 pacientes encaminhados para EDA, com diagnóstico clínico de dispepsia segundo critérios de Roma III. Os laudos endoscópicos foram descritos pelos mesmos endoscopistas para manter o mesmo padrão diagnóstico.

Foram excluídos os pacientes com dificuldade de cognição, aqueles que não concluíram o exame endoscópico, não assinaram o termo de consentimento livre e esclarecido (TCLE) e laudos com informações incompletas e incluídos pacientes do sexo masculino e feminino, que tinham idade maior ou igual a 20 anos e que tinham o diagnóstico prévio de dispepsia.

Os pacientes foram abordados na sala de espera da clínica antes da realização do exame, assinaram o TCLE e responderam a três questionários entregues e explicados pelos entrevistadores: sociodemográfico e clínico com questões abertas e fechadas, que abordavam o nome do paciente, data de nascimento, procedência, gênero, estado civil, cor da pele, grau de instrução e hábitos sociais, além de responderem se já haviam realizado endoscopia previamente e se já possuíam algum diagnóstico endoscópico anterior ou de comorbidades nos demais aparelhos (cardiovascular, respiratório, osteomuscular, endócrino); foi aplicado um questionário de dispepsia funcional contendo os Critérios de ROMA III que tinha como objetivo identificar possíveis queixas de padrão funcional como: dor/desconforto em região epigástrica, incapacidade de terminar uma refeição de tamanho habitual ou empachamento pós prandial, além de pirose em região epigástrica e o período de acometimento dos sintomas. 
As variáveis categóricas foram obtidas por meio de frequência absoluta e relativa percentual e, as variáveis contínuas, através de mediana e intervalo quartil. Realizada análise descritiva e bivariada. A hipótese de independência entre variáveis categóricas foi testada pelos testes Qui-Quadrado de Pearson ou Exato de Fisher e as diferenças entre as medianas aplicou-se o teste de Mann-Whitney. O nível de significância foi 5\%. Utilizou-se o software R Core Team 2020. Estudo aprovado pelo CEP/UNIT, parecer $\mathrm{n}^{\circ} 1.246 .355$.

\section{Resultados}

Foram considerados como dados válidos 847 (98,4\%) laudos. Apresentaram alguma alteração endoscópica 792 (93,5\%) laudos, $36(4,5 \%)$ laudos foram considerados normais, ou seja, sem lesão estrutural e 19 (2,39\%) deles não foram liberados pelos pacientes. A faixa etária mediana (IIQ) foi de 37 (30 a 46) anos, 549 (64,8\%) eram do sexo feminino, 617 (72,8\%) tinham procedência da capital, $419(51 \%)$ se diziam pardos, $470(55,9 \%)$ casados e 440(52,6\%) tinham o ensino médio completo. Quanto aos hábitos de vida, $264(94,6 \%)$ eram etilistas e 39 (14\%) tabagistas (Tabela 1).

Tabela 1. Aspectos sociodemográficos de portadores de sintomas dispépticos submetidos à endoscopia digestiva alta. Aracaju/SE 2018-2019.

\begin{tabular}{|c|c|c|c|c|}
\hline & \multirow[b]{2}{*}{$\begin{array}{c}\text { Dados Válidos } \\
\text { n }(\%)\end{array}$} & \multicolumn{2}{|c|}{ Alterações Endoscópicas } & \multirow[b]{2}{*}{ p-valor } \\
\hline & & $\begin{array}{c}\text { Sim } \\
(n=792)\end{array}$ & $\begin{array}{c}\text { Não } \\
(n=55)\end{array}$ & \\
\hline Idade, Mediana $(I I Q)$ & $847(98,4)$ & $37(30-46)$ & $35(24-42)$ & $0,016^{\mathrm{W}}$ \\
\hline \multicolumn{5}{|l|}{ Procedência, $n(\%)$} \\
\hline Aracaju & $617(72,8)$ & $581(73,4)$ & $36(65,5)$ & \multirow[t]{3}{*}{$0,383^{Q}$} \\
\hline Interior de Sergipe & $121(14,3)$ & $112(14,1)$ & $9(16,4)$ & \\
\hline Outro estado do brasil & $109(12,9)$ & $99(12,5)$ & $10(18,2)$ & \\
\hline \multicolumn{5}{|l|}{ Sexo, $n(\%)$} \\
\hline Feminino & $549(64,8)$ & $505(63,8)$ & $44(80)$ & \multirow[t]{2}{*}{$0,019^{\mathrm{F}}$} \\
\hline Masculino & $298(35,2)$ & $287(36,2)$ & $11(20)$ & \\
\hline \multicolumn{5}{|l|}{ Estado civil, $n(\%)$} \\
\hline Solteiro & $299(35,6)$ & $270(34,3)$ & $29(53,7)$ & \multirow[t]{4}{*}{$0,030^{Q}$} \\
\hline Casado & $470(55,9)$ & $447(56,8)$ & $23(42,6)$ & \\
\hline Viúvo & $18(2,1)$ & $18(2,3)$ & $0(0)$ & \\
\hline Outros & $54(6,4)$ & $52(6,6)$ & $2(3,7)$ & \\
\hline \multicolumn{5}{|l|}{ Cor da pele, $n(\%)$} \\
\hline Branca & $204(24,8)$ & $194(25,3)$ & $10(18,5)$ & \multirow[t]{5}{*}{$0,391 \mathrm{Q}$} \\
\hline Parda & $419(51)$ & $386(50,3)$ & $33(61,1)$ & \\
\hline Preta & $168(20,4)$ & $160(20,8)$ & $8(14,8)$ & \\
\hline Amarela & $29(3,5)$ & $26(3,4)$ & $3(5,6)$ & \\
\hline Indígena & $2(0,2)$ & $2(0,3)$ & $0(0)$ & \\
\hline Álcool, $n(\%)$ & $264(94,6)$ & $247(94,3)$ & $17(100)$ & $0,610^{\mathrm{F}}$ \\
\hline Tabagismo, $n(\%)$ & $39(14)$ & $36(13,7)$ & $3(17,6)$ & $0,715^{\mathrm{F}}$ \\
\hline Drogas ilícitas, $n(\%)$ & $1(0,4)$ & $1(0,4)$ & $0(0)$ & $1,000^{\mathrm{F}}$ \\
\hline \multicolumn{5}{|l|}{ Grau de instrução, $n(\%)$} \\
\hline Analfabeto & $6(0,7)$ & $6(0,8)$ & $0(0)$ & \multirow[t]{2}{*}{$0,987 \mathrm{Q}$} \\
\hline Ensino fundamental incompleto & $32(3,8)$ & $30(3,8)$ & $2(3,8)$ & \\
\hline
\end{tabular}




$\begin{array}{lccc}\text { Ensino fundamental completo } & 97(11,6) & 91(11,6) & 6(11,3) \\ \text { Ensino médio completo } & 440(52,6) & 413(52,7) & 27(50,9) \\ \text { Ensino superior completo } & 262(31,3) & 244(31,1) & 18(34)\end{array}$

Legenda: $\mathrm{n}$ - frequência absoluta. \% - frequência relativa percentual. IIQ - intervalo interquartil. W - Teste de Mann-Whitney. Q - Teste QuiQuadrado de Pearson. F - Teste Exato de Fisher. Fonte: Autores.

Dentre os achados endoscópicos foi estatisticamente significativo o diagnóstico de gastrite (p<0,001), com 661 (89,2) casos. Foram mais prevalentes percentualmente os laudos com diagnóstico de esofagite 168 (22,7\%) e de pólipo 54 (6,4\%). Dos 54 pacientes que apresentaram pólipos, 49 (90,7\%) eram do tipo gástrico, 4 (6,3\%) esofágicos e 3 (3,1\%) duodenais. A presença de úlcera foi identificada em 9 (1,2\%) laudos endoscópicos, sendo localizadas no esôfago, estômago e duodeno. Três tipos de procedimentos cirúrgicos foram detectados: gastroplastia antirrefluxo 1 (6,3\%), fundoplicatura 3 (18,8\%), gastrectomia 12 (75\%). Na categoria “outros” incluíram-se: monilíase esofágica 3 (25\%), estase gástrica 1 (8,3\%), gastropatia hipertensiva 1 $(8,3 \%)$, mucosa gástrica ectópica 3 (25\%), pâncreas ectópico $2(16,2 \%)$, cicatriz duodenal $1(8,3 \%)$ e xantoma duodenal 1 (25\%). Não foi detectado diagnóstico de malignidade na amostra estudada (Tabela 2).

Tabela 2. Achados endoscópicos em pacientes com queixas dispépticas (Aracaju, 2018-2019).

\begin{tabular}{|c|c|c|c|c|}
\hline & \multirow[b]{2}{*}{$\begin{array}{c}\text { Dados Válidos } \\
\mathrm{n}(\%)\end{array}$} & \multicolumn{2}{|c|}{ Alterações Endoscópicas } & \multirow[b]{2}{*}{ p-valor } \\
\hline & & $\begin{array}{c}\text { Sim } \\
\text { n }(\%)\end{array}$ & $\begin{array}{c}\text { Não } \\
\text { n (\%) }\end{array}$ & \\
\hline Realizou endoscopia & $847(98,9)$ & $792(93,5)$ & $55(6,5)$ & $1,000^{\mathrm{F}}$ \\
\hline \multicolumn{5}{|l|}{ Há quanto tempo? } \\
\hline Estou realizando agora & $828(97)$ & $726(97,1)$ & $102(96,2))$ & $0,614^{Q}$ \\
\hline Último mês & $5(0,6)$ & $5(0,7)$ & $0(0)$ & \\
\hline Três meses & $5(0,6)$ & $4(0,5)$ & $1(0,9)$ & \\
\hline Mais de 6 meses & $16(1,9)$ & $13(1,7)$ & $3(2,8)$ & \\
\hline Esofagite & $195(23)$ & $168(22,7)$ & $27(25,7)$ & $0,536^{\mathrm{F}}$ \\
\hline Gastrite & $737(87,1)$ & $661(89,2)$ & $76(72,4)$ & $<0,001^{\mathrm{F}}$ \\
\hline Erosão gástrica & $4(0,5)$ & $4(0,5)$ & $0(0)$ & $1,000^{\mathrm{F}}$ \\
\hline Pólipo & $54(6,4)$ & $52(7)$ & $2(1,9)$ & $0,052^{\mathrm{F}}$ \\
\hline Esofágico & $4(6)$ & $4(6,3)$ & $0(0)$ & $1,000^{\mathrm{F}}$ \\
\hline Gástrico & $51(91,1)$ & $49(90,7)$ & $2(100)$ & $1,000^{\mathrm{F}}$ \\
\hline Duodenal & $3(2,9)$ & $3(3,1)$ & $0(0)$ & $1,000^{\mathrm{F}}$ \\
\hline Duodenite & $20(2,4)$ & $18(2,4)$ & $2(1,9)$ & $1,000^{\mathrm{F}}$ \\
\hline Divertículo Duodenal & $1(1)$ & $1(1)$ & $0(0)$ & $1,000^{\mathrm{F}}$ \\
\hline Outras & $12(1,4)$ & $12(1,41)$ & $0(0)$ & 0,153 \\
\hline Monilíase Esofágica & $3(25)$ & $3(25)$ & $0(0)$ & $1,000^{\mathrm{F}}$ \\
\hline Estase gástrica & $1(8,3)$ & $1(8,3)$ & $0(0)$ & $1,000^{\mathrm{F}}$ \\
\hline Gastropatia Hipertensiva & $1(8,3)$ & $1(8,3)$ & $0(0)$ & $1,000^{\mathrm{F}}$ \\
\hline Mucosa gástrica ectópica & $3(25)$ & $3(25)$ & $0(0)$ & $1,000^{\mathrm{F}}$ \\
\hline Pâncreas ectópico & $2(16,2)$ & $2(16,2)$ & $0(0)$ & $1,000^{\mathrm{F}}$ \\
\hline Cicatriz duodenal & $1(25)$ & $1(25)$ & $0(0)$ & $1,000^{\mathrm{F}}$ \\
\hline Xantoma duodenal & $1(25)$ & $1(25)$ & $0(0)$ & $1,000^{\mathrm{F}}$ \\
\hline Realizou algum procedimento cirúrgico & $19(2,2)$ & $16(2,2)$ & $3(2,9)$ & $0,721^{\mathrm{F}}$ \\
\hline
\end{tabular}




\begin{tabular}{lcccc} 
Gastroplastia antirrefluxo & $1(5,3)$ & $1(6,3)$ & $0(0)$ & $1,000^{\mathrm{F}}$ \\
Fundoplicatura & $4(21,1)$ & $3(18,8)$ & $1(33,3)$ & $0,530^{\mathrm{F}}$ \\
Gastrectomia & $14(73,7)$ & $12(75)$ & $2(66,7)$ & $1,000^{\mathrm{F}}$ \\
Úlcera & $9(1,1)$ & $9(1,2)$ & $0(0)$ & $1,000^{\mathrm{F}}$ \\
\hline
\end{tabular}

Legenda: $\mathrm{n}$ - frequência absoluta. \% - frequência relativa percentual. IIQ - intervalo interquartil. W - Teste de Mann-Whitney.

Q - Teste Qui-Quadrado de Pearson. F - Teste Exato de Fisher. Fonte: Autores.

Quanto a presença de comorbidades entre os participantes do estudo, identificou-se que 341 (43,3\%) respondentes afirmaram possuir algum diagnóstico de doença do tubo digestivo (esôfago, estômago, intestino), 125 (15,9\%) eram portadores de doenças cardiovasculares, 109 (13,9\%) de doenças do aparelho respiratório, 115(14,6\%) doenças do osso, musculoesquelético e tecido conjuntivo, 316 (94\%) tinham diagnóstico de transtorno de ansiedade e 252 (31,9\%) faziam uso de medicamentos.

Dos $92(25,2 \%)$ participantes que relataram ser portadores de doença de esôfago, 31(33,7\%) tinham diagnóstico de esofagite e desses, 30(35,7) tiveram alterações confirmadas pela EDA. Afirmaram ser portadores de doença do refluxo gastroesofágico (DRGE) 57(62\%) respondentes, sendo que em 51(60,7\%) deles foram encontradas alterações estruturais ao exame endoscópico. A gastrite foi citada por 297 (98,7\%) participantes e 278 (98,6\%) tiveram diagnóstico confirmado pela endoscopia. Dentre os 264 (31,2\%) pacientes que afirmaram fazer uso de algum tipo de medicação, foram encontradas alterações endoscópicas em 252 (31,9\%) pacientes (Tabela 3).

Tabela 3. Correlação de achados endoscópicos em pacientes dispépticos portadores de comorbidades. Aracaju, $2018-2019$.

\begin{tabular}{|c|c|c|c|c|}
\hline & \multirow[b]{2}{*}{$\begin{array}{c}\text { Dados válidos } \\
\text { n (\%) }\end{array}$} & \multicolumn{2}{|c|}{ Alteração Endoscópica } & \multirow[b]{2}{*}{ p-valor } \\
\hline & & $\begin{array}{c}\text { Sim } \\
\text { n }(\%)\end{array}$ & $\begin{array}{c}\text { Não } \\
\text { n (\%) }\end{array}$ & \\
\hline Doenças do aparelho cardiovascular (coração) & $130(15,4)$ & $125(15,9)$ & $5(9,3)$ & 0,244 \\
\hline Doenças do aparelho respiratório (pulmão) & $121(14,4)$ & $109(13,9)$ & $12(22,2)$ & 0,107 \\
\hline Doenças do tubo digestivo (esôfago, estômago, intestino) & $365(43,3)$ & $341(43,3)$ & $24(44,4)$ & 0,888 \\
\hline Esôfago & $92(25,2)$ & $84(24,6)$ & $8(33,3)$ & 0,338 \\
\hline Esofagite & $31(33,7)$ & $30(35,7)$ & $1(12,5)$ & 0,259 \\
\hline Hérnia de hiato & $21(22,8)$ & $20(23,8)$ & $1(12,5)$ & 0,677 \\
\hline DRGE & $57(62)$ & $51(60,7)$ & $6(75)$ & 0,706 \\
\hline Pólipos esofágicos & $2(2,2)$ & $2(2,4)$ & $0(0)$ & 1,000 \\
\hline Estômago & $300(82,2)$ & $281(82,4)$ & $19(79,2)$ & 0,782 \\
\hline Gastrite & $297(98,7)$ & $278(98,6)$ & $19(100)$ & 1,000 \\
\hline Pólipos gástricos & $3(1)$ & $3(1,1)$ & $0(0)$ & 1,000 \\
\hline Úlcera gastroduodenal & $10(2,7)$ & $10(2,9)$ & $0(0)$ & 1,000 \\
\hline Intestino & $33(9)$ & $28(8,2)$ & $5(20,8)$ & 0,054 \\
\hline Divertículos & $2(6,1)$ & $2(7,1)$ & $0(0)$ & 1,000 \\
\hline Pólipos duodenais & $10(30,3)$ & $9(32,1)$ & $1(20)$ & 1,000 \\
\hline Retocolite ulcerativa & $2(6,1)$ & $2(7,1)$ & $0(0)$ & 1,000 \\
\hline Síndrome do intestino irritável & $5(15,2)$ & $4(14,3)$ & $1(20)$ & 1,000 \\
\hline Intolerância a lactose & $19(57,6)$ & $16(57,1)$ & $3(60)$ & 1,000 \\
\hline Doença celíaca & $1(3)$ & $1(3,6)$ & $0(0)$ & 1,000 \\
\hline Alergia alimentar & $7(1,9)$ & $6(1,8)$ & $1(4,2)$ & 0,384 \\
\hline Outros & $26(7,1)$ & $26(7,6)$ & $0(0)$ & 0,240 \\
\hline
\end{tabular}




\begin{tabular}{lcccc} 
Duodenite & $2(8)$ & $2(8,3)$ & $0(0)$ & 1,000 \\
Varizes esofágicas & $1(4)$ & $1(4,2)$ & $0(0)$ & 1,000 \\
Esôfago de Barret & $1(4)$ & $1(4,2)$ & $0(0)$ & 1,000 \\
Doenças do fígado, vias biliares e pâncreas & $102(12,1)$ & $97(12,3)$ & $5(9,3)$ & 0,667 \\
Doenças do osso, musculoesquelético e tecido conjuntivo & $121(14,4)$ & $115(14,6)$ & $6(11,1)$ & 0,687 \\
Doenças do sistema endócrino & $86(10,2)$ & $81(10,3)$ & $5(9,3)$ & 1,000 \\
Câncer & $14(1,7)$ & $13(1,7)$ & $1(1,9)$ & 0,610 \\
Depressão & $80(22,1)$ & $77(22,9)$ & $3(11,5)$ & 0,225 \\
Ansiedade & $339(93,6)$ & $316(94)$ & $23(88,5)$ & 0,223 \\
Transtorno do pânico & $24(6,6)$ & $23(6,8)$ & $1(3,8)$ & 1,000 \\
Fobias & $41(11,3)$ & $37(11)$ & $4(15,4)$ & 0,517 \\
Anorexia/bulimia & $2(0,6)$ & $2(0,6)$ & $0(0)$ & 1,000 \\
Insônia & $2(50)$ & $1(33,3)$ & $1(100)$ & 1,000 \\
Enxaqueca & $1(50)$ & $1(100)$ & $0(0)$ & 1,000 \\
Usa medicamento & $264(31,2)$ & $252(31,9)$ & $12(21,8)$ & 0,134 \\
\hline
\end{tabular}

Legenda: $\mathrm{n}$ - frequência absoluta. \% - frequência relativa percentual. Teste Exato de Fisher. Fonte: Autores.

Para identificação de possíveis queixas de padrão funcional, o presente estudo utilizou dos critérios diagnósticos de Roma III para dispepsia funcional correlacionando com pacientes que tiveram alterações vistas à endoscopia para comprovar o padrão de dispepsia de causa orgânica (Tabela 4). A não identificação das queixas a partir da resposta "nunca" foi informada em 279 $(33,4 \%)$ dos dados validos para azia ( $\mathrm{p}<0,085)$, e destes, 256 (32,8\%) laudos tiveram alterações endoscópicas. A partir de 82 $(9,8 \%)$ dos pacientes que responderam a presença de azia todos os dias, 79 (10,1\%) tiveram alterações endoscópicas.

Dado similar foi observado em 320 (38,3\%) dados válidos que responderam como "nunca" para presença de sensação de saciedade após refeição de tamanho habitual nos últimos três meses ( $\mathrm{p}<0,718)$. Foram encontradas alterações endoscópicas em $300(38,5 \%)$ dos laudos. Para 57 (6,9\%) dos dados válidos que informaram não conseguir terminar uma refeição de tamanho habitual, nos últimos 3 meses (p<0,363), 54 (7\%) obtiveram alterações à endoscopia.

Dos 197 (23,5\%) pacientes que referiram dor ou queimação no meio do seu abdome, acima do seu umbigo, e não no peito nos últimos 3 meses ( $<<0,314)$, por mais de um dia por semana, não foram vistas alterações a EDA em apenas $9(16,7 \%)$ deles. Para os 293 (56,1\%) pacientes que informaram que a dor ou queimação já teve duração de 6 meses (p<0,078), $283(57,1 \%)$ tiveram alterações vistas à endoscopia

Tabela 4. Correlação de achados endoscópicos em pacientes dispépticos a partir dos critérios diagnósticos de Roma III para Dispepsia Funcional. Aracaju, 2018-2019.

\begin{tabular}{lccccc}
\hline & & \multicolumn{2}{c}{ Alteração Endoscópica } & \\
\cline { 3 - 4 } & Dados válidos & Sim & Não & p-valor \\
\hline
\end{tabular}

\section{Nos últimos 3 meses, com que frequência você teve dor ou desconforto no meio do seu peito (não relacionada a problemas cardíacos)?}

\section{Nunca}

Menos de um dia por mês

Um dia por mês

Dois a três dias por mês

Um dia por semana

$\begin{array}{rcc}485(57,7) & 459(58,4) & 26(47,3) \\ 48(5,7) & 41(5,2) & 7(12,7) \\ 46(5,5) & 43(5,5) & 3(5,5) \\ 55(6,5) & 52(6,6) & 3(5,5) \\ 35(4,2) & 34(4,3) & 1(1,8) \\ 7 & & \end{array}$


Mais de um dia por semana

Todos os dias

2. Nos últimos 3 meses, com que frequência você teve azia (um desconforto ou dor de queimação no seu peito)?

Nunca

Menos de um dia por mês

Um dia por mês

Dois a três dias por mês

Um dia por semana

Mais de um dia por semana

Todos os dias

3. Nos últimos 3 meses, com que frequência você se sentiu desconfortavelmente cheio (saciado) depois de uma refeição de tamanho habitual?

Nunca

Menos de um dia por mês

Um dia por mês

Dois a três dias por mês

Um dia por semana

Mais de um dia por semana

Todos os dias

4. Você teve esta sensação desconfortável de estar cheio após as refeições por 6 meses?

Não

Sim

5. Nos últimos 3 meses, com que frequência você foi incapaz de terminar uma refeição de tamanho habitual?

Nunca

Menos de um dia por mês

Um dia por mês

Dois a três dias por mês

Um dia por semana

Mais de um dia por semana

Todos os dias

6. Você teve esta incapacidade de terminar

refeições de tamanho habitual por 6 meses ou mais?

Não

Sim

7. Nos últimos 3 meses, com que frequência você teve dor ou queimação no meio do seu abdome, acima do seu umbigo, mas não no seu peito?

Nunca

$\begin{array}{ccc}321(38,3) & 296(37,7) & 25(46,3) \\ 37(4,4) & 33(4,2) & 4(7,4)\end{array}$

$0,085^{Q}$

$\begin{array}{ccc}53(6,3) & 46(5,9) & 7(12,7) \\ 46(5,5) & 42(5,4) & 4(7,3) \\ 96(11,5) & 92(11,8) & 4(7,3) \\ 80(9,6) & 79(10,1) & 1(1,8) \\ 200(23,9) & 187(23,9) & 13(23,6) \\ 82(9,8) & 79(10,1) & 3(5,5)\end{array}$

$\begin{array}{ccc}320(38,3) & 300(38,5) & 20(36,4) \\ 47(5,6) & 44(5,6) & 3(5,5) \\ 37(4,4) & 32(4,1) & 5(9,1) \\ 91(10,9) & 86(11) & 5(9,1) \\ 77(9,2) & 71(9,1) & 6(10,9) \\ 163(19,5) & 152(19,5) & 11(20) \\ 100(12) & 95(12,2) & 5(9,1)\end{array}$

$241(45)$

$219(44)$

$22(59,5)$

$0,086^{\mathrm{F}}$

279 (56)

$15(40,5)$

$\begin{array}{ccc}619(75,4) & 576(75,2) & 43(78,2) \\ 26(3,2) & 24(3,1) & 2(3,6) \\ 29(3,5) & 27(3,5) & 2(3,6) \\ 38(4,6) & 37(4,8) & 1(1,8) \\ 26(3,2) & 26(3,4) & 0(0) \\ 57(6,9) & 54(7) & 3(5,5) \\ 26(3,2) & 22(2,9) & 4(7,3)\end{array}$

$0,363^{Q}$

$\begin{array}{lll}114(50,4) & 108(50,7) & 6(46,2) \\ 112(49,6) & 105(49,3) & 7(53,8)\end{array}$

$0,783^{Q}$ 
Um dia por mês

Dois a três dias por mês

Um dia por semana

Mais de um dia por semana

Todos os dias

8. Você teve esta dor ou queimação por 6 meses ou mais?

Não

Sim

9. Esta dor ou queimação ocorre e depois

desaparece completamente durante o mesmo dia?

Nunca ou raramente

Às vezes

Muitas vezes

Maioria das vezes

Sempre

10. Normalmente, quão severa era a dor ou

queimação no meio do abdome, acima do seu umbigo?

Muito suave

Suave

Moderada

Severa

Muito severa

11. Essa dor ou queimação era aliviada com o uso de antiácidos?

Nunca ou raramente

Às vezes

Muitas vezes

Maioria das vezes

Sempre

Não uso antiácidos

12. Essa dor ou queimação normalmente

melhorava ou passava após a evacuação ou

eliminação de gases?

Nunca ou raramente

Às vezes

Muitas vezes

Maioria das vezes

Sempre

13. Com que frequência essa dor ou desconforto aliviou com movimentos ou trocas de posição do seu corpo?

Nunca ou raramente

Às vezes

$\begin{array}{ccc}61(7,3) & 57(7,3) & 4(7,4) \\ 90(10,7) & 82(10,4) & 8(14,8) \\ 77(9,2) & 74(9,4) & 3(5,6) \\ 197(23,5) & 188(23,9) & 9(16,7) \\ 56(6,7) & 55(7) & 1(1,9)\end{array}$

$\begin{array}{lll}229(43,9) & 212(42,9) & 17(60,7) \\ 293(56,1) & 282(57,1) & 11(39,3)\end{array}$

$0,078^{\mathrm{F}}$

$\begin{array}{ccc}83(15,9) & 79(16,1) & 4(13,3) \\ 127(24,4) & 120(24,4) & 7(23,3) \\ 33(6,3) & 30(6,1) & 3(10) \\ 96(18,4) & 91(18,5) & 5(16,7) \\ 182(34,9) & 171(34,8) & 11(36,7)\end{array}$

$0,927^{\mathrm{Q}}$

$\begin{array}{ccc}14(2,7) & 14(2,9) & 0(0) \\ 121(23,4) & 113(23,1) & 8(27,6) \\ 245(47,3) & 233(47,6) & 12(41,4) \\ 104(20,1) & 97(19,8) & 7(24,1) \\ 34(6,6) & 32(6,5) & 2(6,9)\end{array}$

$\begin{array}{ccc}81(15,6) & 76(15,5) & 5(17,2) \\ 103(19,8) & 100(20,4) & 3(10,3) \\ 29(5,6) & 28(5,7) & 1(3,4) \\ 63(12,1) & 61(12,4) & 2(6,9) \\ 125(24) & 117(23,8) & 8(27,6) \\ 119(22,9) & 109(22,2) & 10(34,5)\end{array}$

$0,507^{\mathrm{Q}}$

$\begin{array}{ccc}271(52,3) & 258(52,8) & 13(44,8) \\ 113(21,8) & 104(21,3) & 9(31) \\ 17(3,3) & 17(3,5) & 0(0) \\ 58(11,2) & 56(11,5) & 2(6,9) \\ 59(11,4) & 54(11) & 5(17,2)\end{array}$

$0,402^{Q}$

$\begin{array}{lll}287(55,2) & 273(55,6) & 14(48,3) \\ 102(19,6) & 96(19,6) & 6(20,7)\end{array}$

0,907 Q 
Muitas vezes

Maioria das vezes

Sempre

14. Nos últimos 6 meses, com que frequência você teve dor constante no meio ou na área superior direita do seu abdome?

Nunca

Menos de um dia por mês

Um dia por mês

Dois a três dias por mês

Um dia por semana

Mais de um dia por semana

Todos os dias

15. Esta dor durou 30 minutos ou mais?

Nunca ou raramente

Às vezes

Muitas vezes

Maioria das vezes

Sempre

16. Essa dor aumentou de intensidade até ficar muito forte e contínua?

Nunca ou raramente

Às vezes

Muitas vezes

Maioria das vezes

Sempre

17. Esta dor desapareceu completamente entre os episódios?

Nunca ou raramente

Às vezes

Muitas vezes

Maioria das vezes

Sempre

18. Essa dor o impediu de realizar suas atividades usuais ou levou-o a ir urgentemente ver um médico ou ir a um serviço de emergência?

Nunca ou raramente

Às vezes

Muitas vezes

Maioria das vezes

Sempre

Dispepsia Funcional Pura

Sim

Não

$\begin{array}{lll}28(5,4) & 26(5,3) & 2(6,9) \\ 50(9,6) & 46(9,4) & 4(13,8) \\ 53(10,2) & 50(10,2) & 3(10,3)\end{array}$

$\begin{array}{ccc}536(64,2) & 494(63,3) & 42(77,8) \\ 39(4,7) & 37(4,7) & 2(3,7) \\ 42(5) & 39(5) & 3(5,6) \\ 54(6,5) & 51(6,5) & 3(5,6) \\ 41(4,9) & 40(5,1) & 1(1,9) \\ 87(10,4) & 85(10,9) & 2(3,7) \\ 36(4,3) & 35(4,5) & 1(1,9)\end{array}$

$99(31,8) \quad 96(32,3) \quad 3(21,4)$

$0,069^{Q}$

$68(21,9) \quad 66(22,2) \quad 2(14,3)$

$19(6,1) \quad 16(5,4) \quad 3(21,4)$

$49(15,8) \quad 45(15,2) \quad 4(28,6)$

$76(24,4) \quad 74(24,9) \quad 2(14,3)$

$\begin{array}{ccc}115(37,2) & 112(37,8) & 3(23,1) \\ 76(24,6) & 70(23,6) & 6(46,2) \\ 33(10,7) & 31(10,5) & 2(15,4) \\ 21(6,8) & 20(6,8) & 1(7,7) \\ 64(20,7) & 63(21,3) & 1(7,7)\end{array}$

$\begin{array}{ccc}72(23,8) & 69(23,8) & 3(23,1) \\ 83(27,4) & 79(27,2) & 4(30,8) \\ 13(4,3) & 13(4,5) & 0(0) \\ 23(7,6) & 22(7,6) & 1(7,7) \\ 112(37) & 107(36,9) & 5(38,5)\end{array}$

$0,981^{Q}$

$\begin{array}{cccc}199(64,6) & 191(64,7) & 8(61,5) & 0,360^{\mathrm{Q}} \\ 66(21,4) & 63(21,4) & 3(23,1) & \\ 14(4,5) & 14(4,7) & 0(0) & \\ 14(4,5) & 14(4,7) & 0(0) & \\ 15(4,9) & 13(4,4) & 2(15,4) & <0,001^{\mathrm{F}} \\ 36(4,3) & 0(0) & 36(65,5) & \end{array}$

Legenda: $\mathrm{n}$ - frequência absoluta. \% - frequência relativa percentual. Q-Teste Qui-Quadrado de Pearson. F-Teste Exato de Fisher. Fonte: Autores. 


\section{Discussão}

A dispepsia pode se manifestar a partir de sintomas relacionados ao aparelho digestório, secundários por doenças orgânicas específicas ou não, como no caso da dispepsia funcional (DF) (Zagari, Law, Fuccio, Pozzato, Forman, \& Bazzoli, 2010). Embora os critérios de Roma III sejam direcionados para doenças funcionais, as quais apresentam uma clínica mais específica em que focam na dor e pirose epigástricas, saciedade precoce e plenitude pós-prandial, deve-se observar os locais anatômicos, através da EDA, para que sejam avaliadas possíveis alterações estruturais que justifiquem esses sintomas.

Estudos citam que Alves (2006), em uma análise retrospectiva com 12.261 relatórios de pacientes que foram submetidos à EDA, identificou que 90,9\% deles possuíam alterações em graus variáveis vistas à endoscopia, achado similar ao obtido por Durães, Fabris, Faraco, Madeira, e Luca (2010) a partir de um estudo descritivo, observacional e retrospectivo, que constatou, em uma amostra com 324 laudos que pertenciam a pacientes portadores de dispepsia, alterações à endoscopia em 96,3\% deles. Mais recentemente, em um estudo transversal, descritivo e analítico, realizado por Fonseca et al. (2014), ao utilizar 450 laudos de pacientes com sintomas dispépticos, também encontrou alterações à EDA em 65,1\% deles. O presente estudo corrobora com tais achados ao demonstrar a presença de alterações endoscópicas em 792 (93,5\%) entrevistados, o que corresponde à maioria dos pacientes com diagnóstico clínico de dispepsia, justificando a etiologia orgânica dos sintomas dispépticos.

O estudo de Fonseca et al. (2014) anteriormente citado demonstrou uma maior prevalência de alterações estruturais em pacientes do sexo feminino e com faixa etária entre 30 a 49 anos. Alves (2006) identificou, também, ser o sexo feminino mais prevalente, e a média de idade no grupo estudado foi em torno de 43,1 anos e mediana de 41 anos. Neste estudo, a idade média encontrada entre os participantes foi de 37 anos e o sexo feminino também foi mais prevalente, o que reitera os achados da literatura.

Observou-se, entretanto, que alguns autores apontaram dados que diferem do que foi encontrado na maior parte da literatura em relação às alterações endoscópicas em portadores de sintomas dispépticos. Johnsen, Bernersen, Straume, Førde, Bostad e Burhol (1991) realizaram um estudo de caso-controle com um grupo de 309 pacientes com queixas dispépticas e 310 controles pareados por idade e sexo. Observaram que 53,5\% deles não apresentaram alterações no exame endoscópico, sendo reconhecido como portador de alguma síndrome funcional. Dados similares também foram observados em um estudo prospectivo feito por Heikkinen, Pikkarainen, Takala, Räsänen, \& Julkunen (1995), com 400 pacientes dispépticos com o objetivo de determinar a prevalência das principais causas de dispepsia. Concluíram que não foram detectadas causas orgânicas para os sintomas relatados em 55\% dos pacientes. Os dados encontrados por estes autores divergem do que foi encontrado no presente estudo, em que apenas 4,5\% tiveram ausência de lesões endoscópicas, o que representa uma minoria de pacientes que possuem uma das condições para definir a dispepsia como funcional.

Piatek-Guziewicz et al. (2014), em estudo retrospectivo realizado com 212 pacientes portadores de dispepsia, constatou que a presença de gastrite, principalmente a eritemato-exsudativa, foi a lesão endoscópica mais comumente encontrada. Durães et al. (2010) analisaram 324 laudos endoscópicos e identificaram que 45,7\% apresentavam gastrite do tipo enantematosa e 29,3\% gastrite endoscópica erosiva como as formas mais frequentes. Em outro estudo prospectivo e transversal, composto por 400 laudos, feito por Chehreh et al. (2016), a gastrite também representou o principal diagnóstico endoscópico em portadores de dispepsia, sendo identificado em $62,5 \%$ dos achados. Neste estudo, os achados foram similares ao evidenciarem que a gastrite foi a doença estrutural mais prevalente dentre as alterações endoscópicas encontradas, representando 98,6\% dos diagnósticos laudados.

Autores citam a esofagite como uma frequente etiologia para justificar os sintomas dispépticos. Heikkinen et al. (1995) avaliaram 400 pacientes dispépticos consecutivamente e observaram a prevalência de esofagite em 15,0\% deles. Thomson et al. (2003), em um estudo multicêntrico com 1.040 pacientes, avaliaram a frequência dos achados endoscópicos e constataram a 
presença de esofagite em $43 \%$ dos pacientes entrevistados. No atual estudo, a esofagite foi diagnosticada em 22,7\%dos laudos analisados, o que elucida a semelhança com os achados da literatura.

O uso de medicamentos, doenças cardíacas e respiratórias, endocrinopatias e outras doenças não pépticas e não digestivas podem ser causas de sintomas dispépticos, o que requer uma indicação precisa da EDA para confirmação e exclusão diagnóstica. (Silva, 2008). Algumas comorbidades não digestivas que podem cursar com alteração dos músculos do trato gastrintestinal promovendo distúrbios motores envolvendo o estômago e o intestino delgado, podem ocasionar retardo do esvaziamento gástrico, tais como doenças reumatológicas, neurológicas, colagenosas, metabólicas entre outras, configuram-se em um estado de gastroparesia (Troncon, 1997). Outras condições consideradas como gastropatias, que provocam lesão da mucosa gástrica com injúria e regeneração epitelial, edema e vasodilatação provocada pelo uso de medicamentos, bebida alcoólica e refluxo biliar podem ser responsáveis por sintomas dispépticos (Genta, 2005).

Considerando que 93,5\% dos laudos de pacientes com dispepsia apresentavam comorbidades associadas, foi possível correlacionar tais condições com os sintomas apresentados. Observou-se que a grande maioria dos portadores de dispepsia apresentavam algum fator orgânico (lesão estrutural) do trato digestório superior e/ou comorbidades extra-digestivas que podiam responder pelos sintomas dispépticos, além de transtornos emocionais como ansiedade, depressão, fobias, transtornos alimentares e outros. Observou-se neste estudo que o exame endoscópico pôde confirmar a etiologia da queixa na maioria dos indivíduos que referiam sintomas dispépticos e tinham alguma comorbidade associada. Afirmaram ser portadores de doença do refluxo gastroesofágico (DRGE) 57 (62\%) respondentes e em 51 (60,7\%) deles foram encontradas alterações estruturais ao exame endoscópico.

Os problemas psicossociais, como a ansiedade ou a depressão, também podem colaborar na apresentação das queixas dispépticas. A produção aumentada de muco está associada a fatores específicos como a ansiedade e o estresse, que irão inibir a ação e produção das células e glândulas responsáveis pela síntese e secreção do muco protetor (Antunes, de Oliveira, Nunes, \& de Oliveira, 2015); (López-Medina, de León, Heredia-Salazar, \& Hernández-Salcedo, 2014). Neste estudo, 316 (94\%) dos portadores de transtorno dispéptico apresentavam algum grau de ansiedade, podendo-se compreender a importância da relação entre ansiedade e estresse emocional e dispepsia.

\section{Conclusão}

A maioria dos pacientes encaminhados para a realização de endoscopia digestiva alta apresentava alguma alteração estrutural compatível com os sintomas dispépticos, enquanto queixa clínica. Entende-se que a frequência de achados endoscópicos em pacientes com tais sintomas merece receber maior atenção, e por isso, estudos como esses são importantes considerando que a dispepsia muitas vezes é tratada como doença crônica não investigada. É possível que a etiologia dos sintomas dispépticos passe despercebida, visto que, o paciente é submetido a tratamento empírico farmacológico, levando a melhora sintomatológica, e, dessa forma, deixa-se de diagnosticar lesões estruturais que justifiquem as queixas dispépticas se de origem orgânica ou funcional. Sugere-se que sejam aplicados, em estudos futuros, questionários que abordem os sintomas gastrointestinais desses pacientes associado à realização do exame de endoscopia digestiva alta para melhor confirmação diagnóstica e conduta.

\section{Referências}

Akram, B., Mohammed, S. S., Mohammed, A. A., \& Obaid, K. A. (2019). Significance of Endoscopyic Findings in Patients with Dyspepsia in Diyala ProvinceIraq Hospital Based Study. Diyala Journal of Medicine, 17(2), 107-114.

Alves, J. S. (2006). Análise dos achados endoscópicos em clínica de endoscopia no período de 1991 a 2001: avaliação crítica da contribuição da endoscopia para diagnóstico do paciente dispéptico: estudo retrospectivo. 
Antunes, M. O., de Oliveira, H. L. A., Nunes, M. E., \& de Oliveira, M. V. M. (2015). Ansiedade e Estresse em Indivíduos Diagnosticados com Gastrite. Revista Bionorte, 4(1), 1-8.

Chehreh, M. G., Shahverdi, E., Khedmat, H., Ghafoorian, A., \& Amini, M. (2016). Endoscopic Findings in Patients with Dyspepsia in Iran. J Dig Dis, 2(3), 211-19.

Durães, E. S. M., Fabris, M. R., Faraco, A. J., Madeira, K., \& Luca, L. R. D. (2010). Análise dos achados endoscópicos em pacientes com dispepsia atendidos no Serviço de Endoscopia do Hospital São João Batista, Criciúma-SC, no período de outubro de 2008 a março de 2009. GED gastroenterol. endosc. dig, 73-78.

Fonseca, A. L. P., D'Acampora, A. J., Zeni, L. B., Sombrio, L. S., \& Oliveira, S. C. V. D. (2014). Análise dos achados endoscópicos mais comuns em pacientes com sintomas dispépticos. GED gastroenterol. endosc. dig, 92-101.

Ford, A. C., Marwaha, A., Sood, R., \& Moayyedi, P. (2015). Global prevalence of, and risk factors for, uninvestigated dyspepsia: a meta-analysis. Gut, 64(7), 1049-1057. https://doi.org/10.1136/gutjnl-2014-307843

Genta, R. M. (2005, November). Differential diagnosis of reactive gastropathy. In Seminars in diagnostic pathology (Vol. 22, No. 4, pp. 273-283). WB Saunders.

Heikkinen, M., Pikkarainen, P., Takala, J., Räsänen, H., \& Julkunen, R. (1995). Etiology of dyspepsia: four hundred unselected consecutive patients in general practice. Scandinavian journal of gastroenterology. 30(6), 519-523. https://doi.org/10.3109/00365529509089783

Johnsen, R., Bernersen, B., Straume, B., Førde, O. H., Bostad, L., \& Burhol, P. G. (1991). Prevalences of endoscopic and histological findings in subjects with and without dyspepsia. British Medical Journal, 302(6779), 749-752.

Koduru, P., Irani, M., \& Quigley, E. (2018). Definition, Pathogenesis, and Management of That Cursed Dyspepsia. Clinical gastroenterology and hepatology: the official clinical practice journal of the American Gastroenterological Association, 16(4), 467-479. https://doi.org/10.1016/j.cgh.2017.09.002

Longstreth, G. F., Lacy, B. E., Talley, N. J., \& Grover, S. (2013). Approach to the adult with dyspepsia.

López-Medina, G., de León, R. C. D., Heredia-Salazar, A. C., \& Hernández-Salcedo, D. R. (2014). Gastric Emphysema a Spectrum of Pneumatosis Intestinalis: A Case Report and Literature Review. Case reports in gastrointestinal medicine, 2014, 891360.

Magalhães, E., de Moraes, G. B. P., \& Braga, M. S. (2018). Abordagem Médica e Psíquica na Dispepsia Funcional-Gastrite Nervosa.

Matsuda, N. M., Maia, C. C., \& Troncon, L. E. D. A. (2010). Dispepsia funcional: revisão de diagnóstico e fisiopatologia. Diagn. tratamento.

Oliveira, S. (2001). Prevalência e fatores associados à dispepsia em adultos na cidade de Pelotas, RS, $1999-2000$ [dissertação]. Pelotas, RS.: Universidade Federal de Pelotas.

Piatek-Guziewicz, A., Przybylska-Feluś, M., Dynowski, W., Zwolińska-Wcisło, M., Lickiewicz, J., \& Mach, T. (2014). Endoscopic and histopathological findings of the upper gastrointestinal tract in patients with functional and organic dyspepsia. Przeglad lekarski, 71(4), 204-209.

Pineda, L. F., Rosas, M. C., Amaya, M. T., Rodríguez, Á., Luque, A., Agudelo, F., \& Sabbagh, L. (2015). Guía de Práctica Clínica para el diagnóstico y tratamiento de la dispepsia en adultos. Revista Colombiana de Gastroenterología, 30(1), 9-16.

Silva, F. M. (2008). Dispepsia: caracterização e abordagem. Revista de Medicina, 87(4), 213-223.

Thomson, A. B., Barkun, A. N., Armstrong, D., Chiba, N., White, R. J., Daniels, S., Escobedo, S., Chakraborty, B., Sinclair, P., \& Van Zanten, S. J. (2003). The prevalence of clinically significant endoscopic findings in primary care patients with uninvestigated dyspepsia: the Canadian Adult Dyspepsia Empiric Treatment - Prompt Endoscopy (CADET-PE) study. Alimentary pharmacology \& therapeutics, 17(12), 1481-1491. https://doi.org/10.1046/j.1365-2036.2003.01646.x

Troncon, L. D. A. (1997). Gastroparesias: revisão de aspectos relacionados ao conceito, à etiopatogenia e ao manejo clínico. Revista da Associação Médica Brasileira, 43(3), 228-236.

Zagari, R. M., Law, G. R., Fuccio, L., Pozzato, P., Forman, D., \& Bazzoli, F. (2010). Dyspeptic symptoms and endoscopic findings in the community: the Loiano-Monghidoro study. The American journal of gastroenterology, 105(3), 565-571. https://doi.org/10.1038/ajg.2009.706 